\title{
Zinc nitride thin films: basic properties and applications
}

\author{
A. Redondo-Cubero ${ }^{a}$, M. Gómez-Castaño ${ }^{a}$, C. García Núñez ${ }^{\mathrm{b}}$, M. Domínguez ${ }^{\mathrm{c}}$, L. Vázquez ${ }^{\mathrm{d}}$ J. L. \\ $\mathrm{Pau}^{\mathrm{a}, *}$ \\ ${ }^{a}$ Grupo de Electrónica y Semiconductores, Facultad de Ciencias, Universidad Autónoma de Madrid,

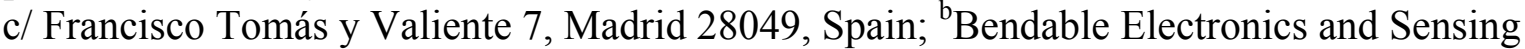 \\ Technologies Group, School of Engineering, University of Glasgow, G12 8QQ, UK; ${ }^{\mathrm{C}}$ Instituto de \\ Ciencias, Benemérita Universidad Autónoma de Puebla, Puebla 72570, México; ${ }^{\mathrm{d}}$ Instituto de \\ Ciencia de Materiales, Consejo Superior de Investigaciones Científicas, E-28049 Madrid, Spain
}

\begin{abstract}
Zinc nitride films can be deposited by radio frequency magnetron sputtering using a $\mathrm{Zn}$ target at substrate temperatures lower than $250{ }^{\circ} \mathrm{C}$. This low deposition temperature makes the material compatible with flexible substrates. The asgrown layers present a black color, polycrystalline structures, large conductivities, and large visible light absorption. Different studies have reported about the severe oxidation of the layers in ambient conditions. Different compositional, structural and optical characterization techniques have shown that the films turn into $\mathrm{ZnO}$ polycrystalline layers, showing visible transparency and semi-insulating properties after total transformation. The oxidation rate is fairly constant as a function of time and depends on environmental parameters such as relative humidity or temperature. Taking advantage of those properties, potential applications of zinc nitride films in environmental sensing have been studied in the recent years. This work reviews the state-of-the-art of the zinc nitride technology and the development of several devices such as humidity indicators, thin film (photo)transistors and sweat monitoring sensors.
\end{abstract}

Keywords: zinc nitride, thin film transistors, humidity sensors, perspiration sensors

\section{INTRODUCTION}

Zinc nitride $\left(\mathrm{Zn}_{3} \mathrm{~N}_{2}\right)$ is a scarcely known II-V compound that crystallizes in the anti-bixbyite structure with a body centered cubic lattice. ${ }^{1}$ It presents n-type conductivity due to the substitutional incorporation of oxygen atoms in nitrogen sites. Carrier concentrations in the $10^{18}-10^{19} \mathrm{~cm}^{-3}$ range have been measured by Hall effect in undoped samples grown by magnetron sputtering and molecular beam epitaxy.,3 Thin films deposited by radio frequency sputtering present good electrical properties such as high mobility and conductivities in the $10^{-2}-10^{-3} \Omega \mathrm{cm}$ range. A recent report shows that the material has a very small electron effective mass (Table 1), which could benefit the intergrain transport in polycrystalline layers. $^{4}$

However, one of the main reasons why this material has not drawn the attention of the scientific community is its poor stability in ambient conditions. Studies have demonstrated that it easily reacts with the ambient gases leading to the formation of $\mathrm{ZnO}$ wurtzite cyrstals. ${ }^{5}$ Besides complicating the development of applications around a metastable material, its high reactivity has hindered the accurate determination of the bandgap energy and the optical constants. The asdeposited bandgap energy of $\mathrm{Zn}_{3} \mathrm{~N}_{2}$ thin films grown at room temperature using a pure nitrogen plasma is around 1.46 $\mathrm{eV}$, although its effective value reduces to $1.25 \mathrm{eV}$ as the deposition temperature increases. ${ }^{2}$ Higher values have been reported through the use of other plasma gas mixtures including $\mathrm{Ar}$ and $\mathrm{O}_{2} .{ }^{6}$ Furthermore, studies performed in samples deposited by magnetron sputtering showed that the apparent bandgap energy tends to blue shift with time, producing an increase of the transparency to visible light. A careful ellipsometric analysis of $\mathrm{Zn}_{3} \mathrm{~N}_{2}$ layers allowed to extract the optical constants, free from artifact effects induced by the unintentional oxidation. ${ }^{7}$ The same technique revealed that the oxidation rate is fairly constant for stable ambient conditions. Thus, the thickness of the oxide formed on top of the nitride layer increases linearly with time. This behavior is typical in oxide layers that contain pores and grain boundaries through which oxygen diffuses to reach the interface with the nitride layer. ${ }^{8}$ The defects come out as a relaxation mechanism due to different density and structure of $\mathrm{ZnO}$ and $\mathrm{Zn}_{3} \mathrm{~N}_{2}$.

Oxide-based Materials and Devices VIII, edited by Ferechteh H. Teherani, David C. Look, David J. Rogers, Ivan Bozovic. Proc. of SPIE Vol. 10105, 101051B · @ 2017 SPIE CCC code: $0277-786 X / 17 / \$ 18 \cdot$ doi: $10.1117 / 12.2253044$ 
The high reactivity of $\mathrm{Zn}_{3} \mathrm{~N}_{2}$ is particularly interesting in sensing applications. Environmental parameters such as the temperature or the relative humidity have a strong influence on the oxidation rate. Furthermore, the nitride oxidation speeds up in aqueous media where the nitride reacts with water molecules to yield $\mathrm{ZnO}$ and ammonia according to the following reaction

$$
\mathrm{Zn}_{3} \mathrm{~N}_{2}+3 \mathrm{H}_{2} \mathrm{O} \rightarrow 3 \mathrm{ZnO}+2 \mathrm{NH}_{3}
$$

On the other hand, the use of capping layers helps to reduce significantly the oxidation rate enabling the development of applications that can take advantage of their good electronic properties. Thus, $\mathrm{ZnO}$ thin films in-situ deposited after the nitride growth have been used to passivate the nitride surface allowing the use of standard processing steps such as organic cleaning, photolithography or photoresist lift-off. The introduction of this capping layer has enabled the fabrication of thin film transistors including several processing steps.

In this paper we will review our last investigations about the conditions that lead to the nitride oxidation and new applications recently developed to exploit the material properties.

Table 1. Basic properties of $\mathrm{Zn}_{3} \mathrm{~N}_{2}$.

\begin{tabular}{|ll|}
\hline Crystal structure & anti-bixbyite ( Ia $\overline{3}$ space group $)^{9}$ \\
Lattice parameter & $9.769 \AA^{10}$ \\
Energy bandgap & $1.25-1.46 \mathrm{eV}$ (direct) \\
Density & $6.22 \mathrm{~g} / \mathrm{cm}^{3}$ \\
Mobility & $100-350 \mathrm{~cm}^{2} / \mathrm{V} \mathrm{s}$ \\
Carrier concentrations & $10^{18}-10^{20} \mathrm{~cm}^{-3}$ \\
Refractive index (n) & $2.5(@ 2.0 \mathrm{eV})$ \\
Extinction coefficient (k) & $0.7(@ 2.0 \mathrm{eV})$ \\
Electron effective mass & $0.08 \pm 0.03 \mathrm{~m}_{0}^{4}$ \\
\hline
\end{tabular}

\section{HUMIDITY EFFECTS ON THE OXIDATION RATE}

The effects of humidity on the oxidation rate were investigated in ref. 11. In that work, a device was built based on the deposition of aluminum coplanar contacts on the surface of zinc nitride films deposited on glass substrates. Through the use of a calibrated HIH-4000 relative humidity sensor, measurements of the electrical resistance between contacts were taken at room temperature while controlling an ultrasonic humidifier to keep a steady relative humidity. After a few days, the resistivity increases abruptly from the original resistance as the oxidation progresses (Figure 1(a)). This abrupt resistivity change occurs when the oxide growth starts to impact strongly on the electrical conduction through the nitride layer. The experiments also demonstrated that the onset of the resistivity increase depends on the temperature and relative humidity ( $\xi$ ) conditions. Thus, the storage at $\xi=40 \%$ produces a faster increase than the storage at $\xi=25 \%$. The comparison of two samples stored at two different temperatures $\left(20\right.$ and $\left.50{ }^{\circ} \mathrm{C}\right)$ under the same relative humidity shows that the temperature is another critical factor to change the oxidation behavior. Thus, the higher temperature produces a longer onset of the resistivity enhancement. The slope of the resistivity increase is also dependent on the relative humidity. Figure 1(b) plots the resistivity as a function of time for a sample kept in air ambient and a sample kept in water between measurements. The slope is almost two orders of magnitude higher in the water sample than in the air sample.

The X-ray diffraction showed that the prolonged exposure in highly humid ambient leads to the formation of $\mathrm{ZnO}$ crystals and a reduction of the $\mathrm{Zn}_{3} \mathrm{~N}_{2}$ material. Furthermore, scanning electron and atomic force microscopy images indicated that the surface roughness increases as a result of the oxidation and that the grain size of the $\mathrm{ZnO}$ formed on top is significantly lower than the grain size of the nitride film. Time of flight measurements in ion beam analysis and spectroscopic ellipsometry confirmed that the oxide layer becomes thicker as the oxidation progresses likely fed by the oxygen diffusion through grain boundaries. The thickness of the layer increases linearly with time following the typical behavior found in defective oxide layers. 
The oxidation also provokes the increase of the visible transmittance, as shown in Figure 2. The transmittance in the visible range increases notably with the oxidation as the nitride thickness diminishes. As in the resistivity case, the relative humidity plays an important role in the transformation time. Unless the surface is passivated, the oxidation process ends up transforming the full nitride film into an oxide layer with a very high visible transparency.

The oxidation time, defined as the time required to fully transform the nitride layer into an oxide layer, varies from a few hours to several days depending on the ambient conditions. That time can be also adjusted by changing the thickness of the nitride layer. Since the oxide growth rate is approximately constant along the transformation process, the oxidation time scales linearly with the nitride thickness. This property enables the development of humidity indicators with a tailorable maximum humidity dose. The details of this novel technology are given in the following section.

(a)

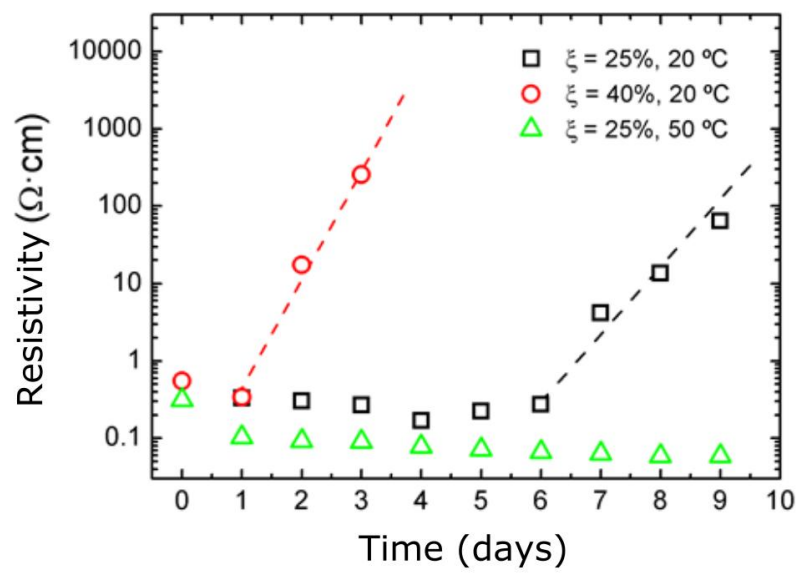

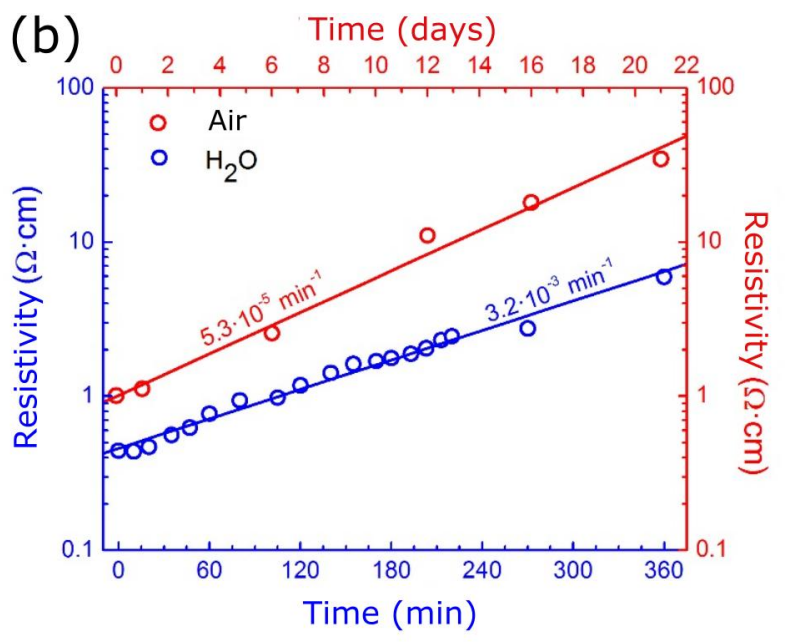

Figure 1. (a) Resistivity as a function of time for three samples stored in air ambient at different relative humidity (25 and $40 \%$ ) and temperature $\left(20\right.$ and $\left.50^{\circ} \mathrm{C}\right)$. (b) Comparison of the resistivity enhancement for samples stored in air (red) and water (blue). Water data are linked to the left and bottom axis of the figure whereas air data coordinates are found in right and top axis.

(a)

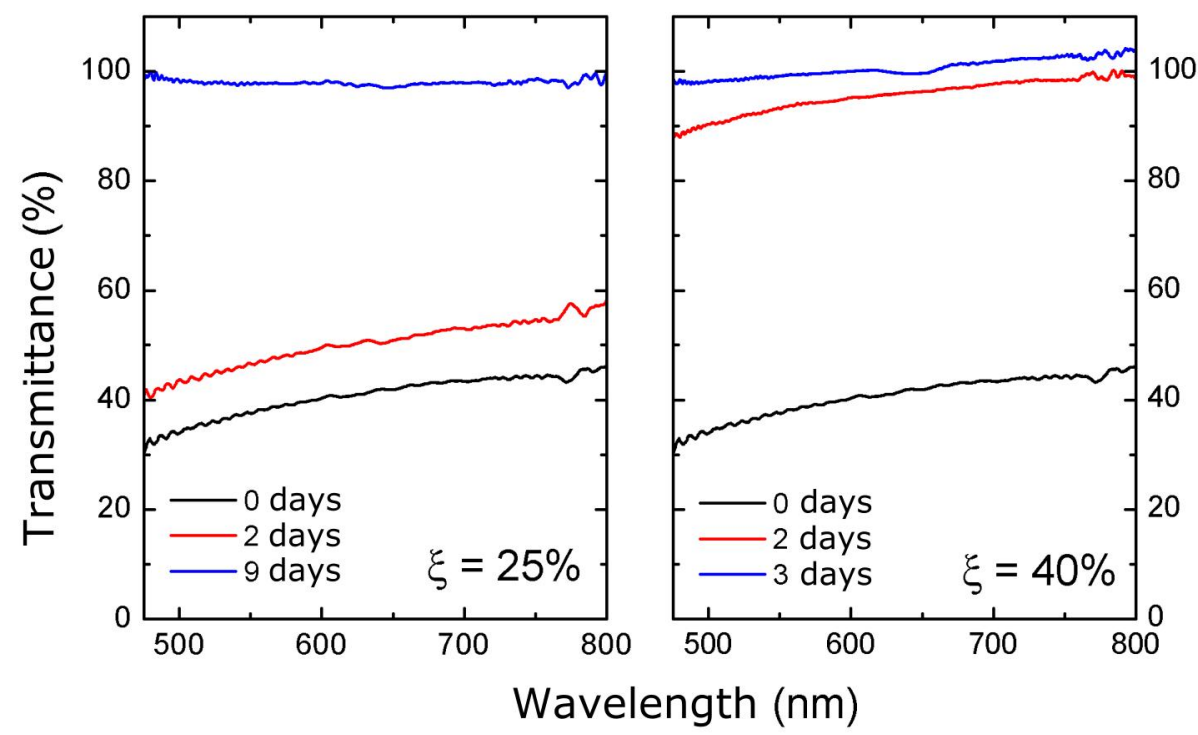

(b) 00 0 0 40 0 


\section{APPLICATIONS}

\subsection{Thin film transistors}

The high mobility measured at large carrier concentrations is a promising characteristic for the fabrication of thin film transistors based on zinc nitride. The small effective mass seems to be behind the large electron mobilities found in this material. ${ }^{4}$ Several reports have shown transistor performance using top-gated and bottom-gated configurations. ${ }^{12,13}$ The bottom-gated devices also exhibited photoconductivity when the devices were illuminated with visible/infrared light. On the other hand, large photoconductive gains were obtained in top-gate transistors. Since nitride films with high electrical conductivity can be deposited at room temperature, the fabrication of devices on flexible substrates is an excellent application where the nitride transistors may have a strong impact.

One of the critical points for the development of those devices has been the use of a capping layer on the nitride surface that enables the fabrication of devices with a reduced impact from the chemicals employed during the photolithographic process. Although further investigations are still needed to improve the stability of the devices in the long run, the in-situ deposition of $\mathrm{ZnO}$ seems to be the best solution found in the literature for the moment to passivate the surface.

\subsection{Humidity indicators}

Following the studies on the effects of humidity on $\mathrm{Zn}_{3} \mathrm{~N}_{2}$ thin films, humidity indicators have been developed during the past year and protected through patent application. These indicators consist of nitride thin films deposited on polycarbonate or polyethylene substrates that can be attached to paper cards for the control of ambient conditions during the transport of goods (Figure 3). The characteristics of the indicators are non-reversible preventing possible misconduct and unethical behavior during the transportation of merchandise by land, by air or by water. The maximum humidity that leads to the transformation can be tailored by changing the thickness of the layer. Thus, the indicators can be adapted to a large number of products depending on their sensitivity to highly humid environments. Unlike current commercial indicators, these devices are free of chloride compounds and free of cobalt which make them more environmentally friendly, fulfilling the European directives and reducing the hazardous risks of the manufacturer employees.

The indicators can be read visually or electronically by simple means, and they can be connected to radio-frequency identification devices to track the conditions of the goods in real time.

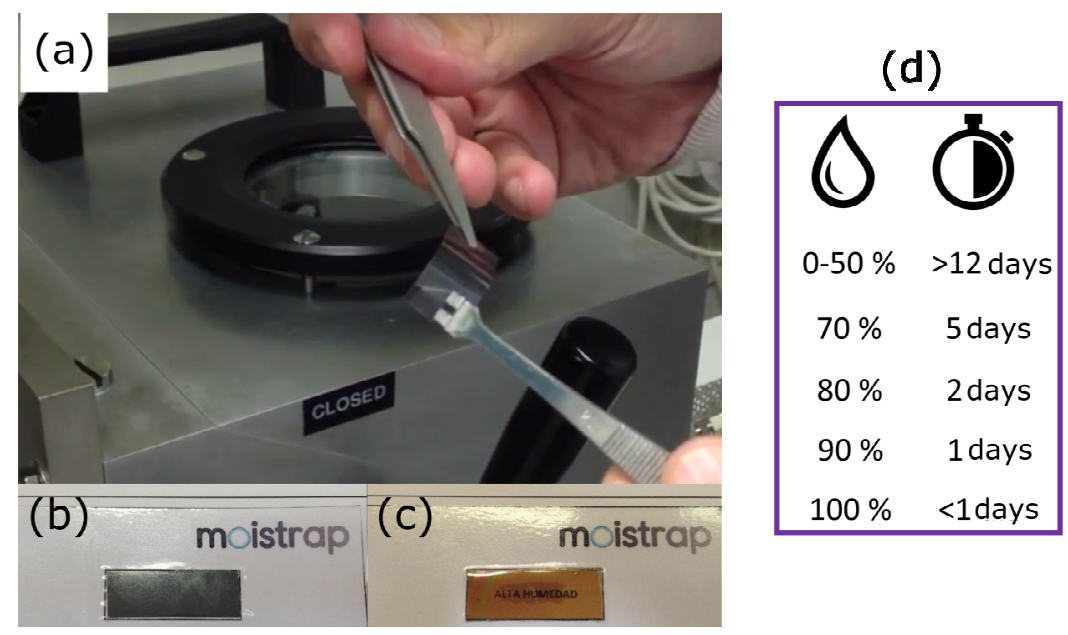

Figure 3. (a) Picture of the zinc nitride films prepared on flexible substrates after deposition. Humidity indicators before (b) and after (c) transformation. (d) Table of values for the oxidation time as a function of the relative humidity. 


\subsection{Perspiration sensors}

The high surface reactivity of zinc nitride can be exploited in sensing applications for biomedical purposes. The resistance of the thin films is sensitive to the surface charge on the surface. Commonly, the sweat contains negative ions that are tugged out by the positively charged sweat pores to avoid excess heat and keep the human body refrigerated. ${ }^{14}$ The sweat vapor can condensate on a cold surface producing a negative charge sheet.

A zinc nitride device has been developed to monitor the production of human sweat. The device comprises two electrodes on the surface of the zinc nitride film. It is protected with a filter bag to prevent from excess exposure somewhat that may limit its response speed. It can be hosted on any part of the body underneath the clothes by using simple double-tape adhesive.

Figure 4 shows the response of such device during a moderate workout session of 25 min (Iexercise) in comparison to the signal obtained during a 1-hour nap (Siesta). The voltage drop across the contacts (y-axis) reduces in both cases but it does it more significantly during the workout session. The baseline for both cases is indicated using dashed lines. After removing the device from the measurement region, the original signal is recovered in a few minutes.
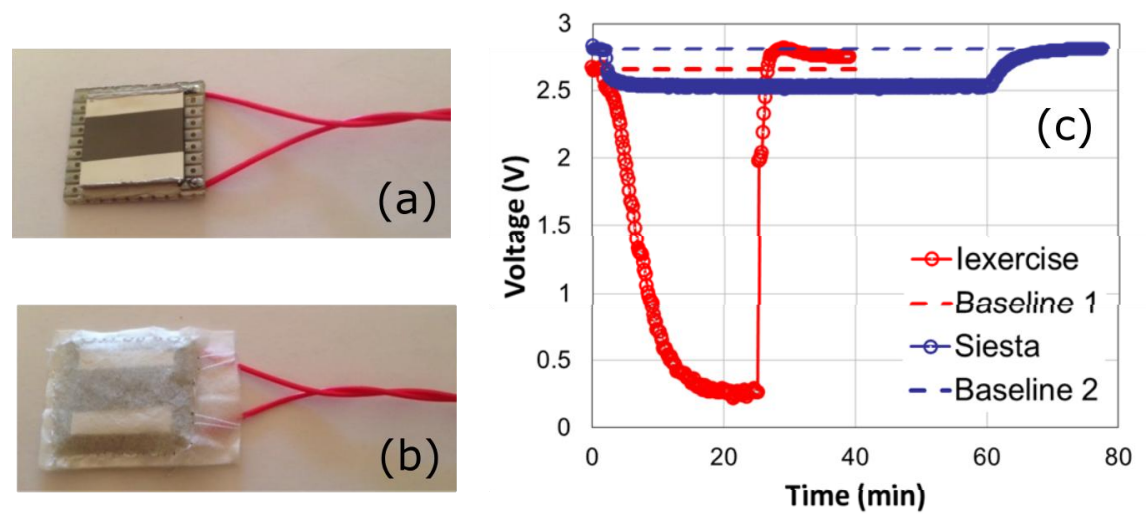

Figure 4. (a) Sweat sensors fabricated from the deposition of metallic contacts on a $\mathrm{Zn}_{3} \mathrm{~N}_{2}$ thin film. (b) The same sweat sensor after covering it with a filter bag to improve the device performance at large perspiration conditions. (c) Voltage drop across the contacts in a simple voltage divider setup connected to a $5 \mathrm{~V}$ power supply.

\section{CONCLUSIONS}

Thanks to its high conductivity, its semiconductor properties and its low deposition temperatures, $\mathrm{Zn}_{3} \mathrm{~N}_{2}$ thin films have demonstrated to be good candidates for the development of different sensing devices. Its metastability can be exploited in humidity sensing applications where the strong difference between the properties of the nitride film and those of the resultant oxide layer is a clear advantage. The successful surface passivation and the characteristics found in thin film transistors may also open new possibilities for this material in flexible electronics.

\section{ACKNOWLEDGMENTS}

We thank Eduardo Ruiz for technical help during the sample preparation. This research is supported by the MINECO (CTQ2014-53334-C2-2-R) and Comunidad de Madrid (NANOAVANSENS ref. S2013/MIT-3029). ARC acknowledges Ramón y Cajal program (under contract number RYC-2015-18047).

\section{REFERENCES}

[1] Suda, T. and Kakishita, K., "Band-gap energy and electron effective mass of polycrystalline $\mathrm{Zn}_{3} \mathrm{~N}_{2}$," J. Appl. Phys. 99(7), 076101 (2006).

[2] García Núñez, C., Pau, J. L., Hernández, M. J., Cervera, M., Ruiz, E., and Piqueras, J., "On the zinc nitride properties and the unintentional incorporation of oxygen," Thin Solid Films 520, 1924-1929 (2012). 
[3] Wu, P., Tiedje, T., Alimohammadi, H., Bahrami-Yekta, V., Masnadi-Shirazi, M., and Wang, C., "Molecular beam epitaxy growth and optical properties of single crystal $\mathrm{Zn}_{3} \mathrm{~N}_{2}$ films," Semicond. Sci. Technol. 31(10), 10LT01 (2016).

[4] Cao, X., Yamaguchi, Y., Ninomiya, Y., and Yamada, N., "Comparative study of electron transport mechanisms in epitaxial and polycrystalline zinc nitride films," J. Appl. Phys. 119(2), 025104 (2016).

[5] García Núñez, C., Pau, J. L., Hernández, M. J., Cervera, M., Ruíz, E., and Piqueras, J., "Influence of air exposure on the compositional nature of $\mathrm{Zn}_{3} \mathrm{~N}_{2}$ thin films," Thin Solid Films 522, 208-211 (2012).

[6] Cao, X., Sato, A., Ninomiya, Y., and Yamada, N., "Oxygen-Doped Zinc Nitride as a High-Mobility NitrideBased Semiconductor," J. Phys. Chem. C 119 (10), 5327-5333 (2015).

[7] García Núñez, C., Pau, J. L., Hernández, M. J., Cervera, M., and Piqueras, J., "On the true optical constants properties of zinc nitride," Appl. Phys. Lett. 99, 232112 (2011).

[8] Fehlner, F. P., [Low-temperature oxidation: the role of vitreous oxides], Wiley-Interscience, New York (1986).

[9] Yoo, S.-H., Walsh, A., Scanlon, D. O., and Soon, A., "Electronic structure and band alignment of zinc nitride, $\mathrm{Zn}_{3} \mathrm{~N}_{2}$," RSC Adv. 4, 3306-3311 (2014).

[10] Partin, D. E., Williams, D. J., and O'Keeffe, M., "The crystal structures of $\mathrm{Mg}_{3} \mathrm{~N}_{2}$ and $\mathrm{Zn}_{3} \mathrm{~N}_{2}$," J. Solid State Chem., 132(1), 56-59 (1997).

[11] Gómez-Castaño, M., Redondo-Cubero, A., Vázquez, L., and Pau, J. L., "Analysis of zinc nitride resistive indicators under different relative humidity conditions," ACS Appl. Mater. Interfaces 8(42), 29163-29168 (2016).

[12] García Núñez, C., Pau, J. L., Ruiz, E., and Piqueras, J., "Thin film transistors based on zinc nitride as a channel layer for optoelectronic devices," Appl. Phys. Lett. 101(25), 253501 (2012).

[13] Domínguez, M. A., Pau, J. L., Gómez-Castaño, M., Luna-López, J. A., and Rosales, P., "High mobility thin film transistors based on zinc nitride deposited at room temperature," Thin Solid Films 619, 261-264 (2016).

[14] Jessen, C., [Temperature regulation in humans and other mammals], Springer, Berlin (2000). 